\title{
College students' communication about complementary and alternative medicine practices
}

\author{
Heather J. Carmack, ${ }^{1}$ Julie A. Serafin ${ }^{2}$ \\ ${ }^{1}$ Department of Communication Studies, University of Alabama, Tuscaloosa, AL; ${ }^{2}$ PRA Health Sciences, Raleigh, NC, USA
}

\begin{abstract}
While the use of Complementary/Alternative Medicine (CAM) has increased over the last two decades, how patients communicate about their decisions to use CAM has yet to be fully explored. The purpose of this study is to examine college students' decision-making and communication about CAM. Fourteen college students participated in semi-structured focus group interviews about their CAM use and communication about CAM with family, friends, and health care providers. Participants use CAM to improve their physical and mental health. However, they have difficulties justifying their decision to practice CAM to others. They relied on CAM not only for physical and mental stability, but also as a sounding board for larger spiritual issues. Participants use passive communication strategies to communicate about their CAM use and often do not disclose CAM use to their providers because of the stigmas related to CAM. There is potential danger for CAM users who do not disclose CAM use to providers, family, and friends. More efforts are needed to encourage open and honest communication about CAM use.
\end{abstract}

\section{Introduction}

Internationally, the biomedical health model has been the primary methodology for health care treatment. ${ }^{1}$ Within the last ten years, however, Complementary/Alternative Medicine (CAM), a group of diverse medical and healthcare systems, practices, and products that are

Correspondence: Heather J. Carmack, Department of Communication Studies, University of Alabama, PO Box 87072, Tuscaloosa, AL 35487, USA.

Tel.: 740.438 .0469

E-mail: heather.carmack1@gmail.com

Key words: College students; Communication; Complementary and alternative medicine; Patient perspective.

Contributions: HC, data collection and analysis, manuscript writeup, manuscript editing; JS, research assistant, data collection and analysis, literature review, draft write-up.

Conflict of interest: the authors declare no potential conflict of interest.

Funding: None.

Received for publication: 25 October 2017.

Revision received: 21 March 2018.

Accepted for publication: 9 April 2018.

This work is licensed under a Creative Commons Attribution NonCommercial 4.0 License (CC BY-NC 4.0).

CCopyright H.J. Carmack and J.A. Serafin, 2018

Licensee PAGEPress, Italy

Qualitative Research in Medicine \& Healthcare 2018; 2:14-19

doi:10.4081/qrmh.2018.7161 not presently considered part of conventional medicine, has been gaining momentum among patients and health care practitioners. ${ }^{2}$ CAM refers to a wide range of ethnocultural therapeutic practices such as aromatherapy, herbalism, and Reiki. ${ }^{1}$ The introduction of CAM modalities in biomedical treatment has been gradual, ${ }^{3}$ in part because of the perception among biomedical providers that unconventional treatments represents a rejection of and challenge to the biomedical approach, their lack of general knowledge about CAM, and unproven scientific evidence of their efficacy.,

Research on communication and CAM suggests that patients are hesitant to talk with their providers about CAM because they fear providers will stop the conversation. ${ }^{6}$ Patients' perception of provider rejection or indifference to CAM can strain patient-provider communication. ${ }^{7}$ To address their needs, some patients go directly to CAM providers. CAM specialists practice the field of integrative medicine, which reaffirms the importance of the relationship between practitioners and patients. ${ }^{7}$ Previous research suggests that increased provider education of CAM use and proper integration of CAM and biomedical practices can improve patient-provider communication about $\mathrm{CAM}^{8}$

Incorporating CAM therapies into the conventional health system could possibly contribute to a wide cultural shift in contemporary healthcare. ${ }^{9}$ One demographic group who generally supports this health culture shift is college students. As early adopters, college students are a unique population who are open to and likely to use CAM. ${ }^{10}$ Many students who actively use CAM view it as a treatment for illnesses, as well as a promotion of health and vitality. ${ }^{11}$ College students already report using CAM therapies such as multivitamins, yoga, massage therapy, mediation, and spiritual healing as resources to improve 
their health. ${ }^{12}$ College can also be very stressful due to workload as well as balancing social, educational and health demands. Unfortunately due to these high levels of stress, college students are at a higher risk for feelings of anxiety and depression than the general population. ${ }^{13} \mathrm{Anx}-$ iety, fatigue, insomnia, and chronic pain have been motivating factors of CAM use among college students ${ }^{13}$ and are used to treat depression and anxiety. ${ }^{14}$ This can create a pathway for student CAM users to explore alternative health options to address their spiritual, physical, and mental needs. These studies identified what CAM therapies students are using, but they do not talk about the challenges students experience when communicating about their CAM choices with others, including family, friends, and providers. This study examines college students' communication about their CAM use decisions.

\section{Literature review}

Since the late 1990s, integrating complementary and alternative medicine with traditional Western medicine has been a struggle. ${ }^{15}$ As the dominant approach to health care, the biomedical model traditionally tends to treat patients' physical illnesses without considering other components of human health, namely mental, spiritual, and emotional. Many patients are now focusing on treating these areas along with their physical ailments, turning to CAM therapies to complement traditional ones. These alternative practices have been used to promote physical, mental, and spiritual health. ${ }^{16}$ Although health care providers realize they are not scientifically proven, many professionals who work with complementary medicine promote CAM for additional health benefits. ${ }^{16}$

CAM therapies look to treat patients as integrated whole beings rather than focusing on treating one part of the body. ${ }^{7}$ The National Center for Complementary and Alternative Medicine classifies CAM therapies into four sectors: natural products and supplements, mind-body interventions, manipulations and body-based methods, and energy therapies. ${ }^{7}$ The first classification of CAM therapies is centered on supplements and natural remedies such as herbal medicines, vitamins, and minerals. Natural remedies and supplements have also been recognized as dietary supplements that are consumed to boost the immune system, add energy, and aid in the balance of an individual's diet. ${ }^{7}$ Mind-body interventions include practices such as meditations, yoga, acupuncture, prayer, breathing exercises, and hypnotherapy. ${ }^{7}$ This CAM category focuses specifically on mental and spiritual health that typically is overlooked in the dominant biomedical health approach. Mind-body practices are also common techniques to reduce stress, anxiety, chronic pain, and mood disorders. ${ }^{17}$ Manipulation and body-based methods, such as massage therapy and chiropractic manipulation, focus on physical aspects of health just like the biomedical model; however, they are considered additive techniques that help with a patient's realignment of the body. ${ }^{17}$
The fourth of the CAM treatments, energy therapies, includes magnet therapy, Reiki, and healing touch. ${ }^{7}$ Energy therapies are generally utilized by patients to reduce anxiety, reduce fatigue, and improve their quality of life. ${ }^{16} \mathrm{Al}-$ though these CAM techniques are all considered body-based therapies, they serve and treat multiple forms of physical and mental health issues. ${ }^{7}$

\section{Communication and complementary/alternative medicine}

College students' use of CAM therapies such as aromatherapy (use of essential oils), meditation, and acupuncture to treat depression, anxiety, insomnia, and chronic pain are well documented. ${ }^{13-14}$ However, the use of CAM and holistic medicine continues to be recognized only as a secondary form of medical treatment. ${ }^{18}$ The lack of awareness of complementary and alternative medicine in the dominant health care field is due to many health care providers' deficient knowledge on this subject. ${ }^{19}$ Admittedly, biomedical practitioners often state they have limited knowledge about CAM therapies. ${ }^{6}$ Consequently, this can create ambiguity for patients who want to discuss CAM options with their health care providers.

Another major concern causing health care providers to dismiss the credibility of CAM is the lack of scientific evidence proving this type of therapy can significantly treat illness. ${ }^{7}$ One of the main reasons allopathic health care providers steer away from this topic is because there is a lack of scientific research about CAM health benefits and efficacy. ${ }^{6}$ Providers may not offer these alternative treatments to their patients due to the unknown health risks. Many health care providers and clinicians believe CAM therapies represent a rejection and challenge to mainstream medication and treatment. ${ }^{18}$ For those providers who do have a general knowledge of CAM therapies, studies have shown they are inconsistent in discussing the benefits with their patients. ${ }^{6}$ Regardless, there are additional factors that prevent the awareness of CAM into the mainstream health care community. Many patients state they are not only wary to discuss CAM therapies with their biomedical providers, but time limits during appointments prevent the discussion regarding CAM usage. ${ }^{7}$ Patients have indicated they fear disapproval from their biomedical providers if they reference CAM therapies as a form of treating illness and disease. ${ }^{20}$

Another factor that contributes to the lack of information regarding CAM therapies is the type of communication medium in which the information is disseminated. Many patients learn about CAM therapies through conversations with friends and family or through their own personal research..$^{21}$ Patients often turn to the Internet or lay publications to read about the benefits of CAM. ${ }^{21-22}$ These sources, especially lay publications and pamphlets, discuss holistic and allopathic medicine and how they can successfully be integrated. ${ }^{22}$ One of the concerns with these search methods is the absence of credibility about 
CAM information found on the Internet. Word of mouth with family and friends can also be biased due to their lack of scientific knowledge of CAM. ${ }^{7}$

To bridge the gap of communication regarding CAM use in the traditional health care system, some patients go directly to CAM providers who practice integrative medicine, which focuses on a wide range of health including mental, physical and spiritual well-being. ${ }^{7}$ The increase of face-to-face communication with CAM providers establishes and reinforces credibility of CAM and the providers as knowledgeable providers who can express positive attitudes of its health benefits. ${ }^{7}$ Because of this, patients are more likely to support CAM's professional and therapeutic legitimacy. ${ }^{1}$ Patients may also find a CAM advocate from other allopathic providers. For example, nurses often serve as the intermediate communicator between conventional provider and patients. ${ }^{1}$ This may be because nurses tend to be more patient-centered as opposed to biomedical doctors, who are disease-centered. ${ }^{1}$

Multiple ways CAM practitioners are increasing mindfulness include improving health care awareness by investment in relationship-centered care, understanding holism, and promoting self-reflection, and self-care. ${ }^{8}$ These communication solutions will help to create mutual understanding and patient-provider dialogue regarding CAM usage, increase individuals' comprehension of CAM therapies, and promote individuals to take action in prevention of illness by incorporating these therapies into their pre-existing health practices. Based on the literature, we asked the following research questions:

RQ1: What are college students' primary reasons

for using CAM therapies?

RQ2: How do students communicate their CAM

practices to others?

RQ3: How do students make sense of others' responses to their use of CAM?

\section{Methods}

Fourteen students from a large Mid-Atlantic university participated in focus group interviews: 11 females (78.6\%) and three males (21.4\%). Ages ranged from 18 to 22 years $(M=20)$. Data collection began once the authors received university IRB approval. Students were primarily recruited through a research participant pool and word of mouth about the study. Two participants identified as freshman, one as a sophomore, nine as juniors, and two as seniors. Participants reported using a variety of CAM therapies, including yoga, meditation, acupuncture, natural oils, and aromatherapy. Nine participants reported using CAM therapies daily, three used it weekly, and two used it monthly.

The researchers conducted three focus groups $(M=4)$. The researchers used a semi-structured interview protocol with questions pertaining to why college students use CAM, perceived health benefits of CAM, students' com- munication about CAM with others, and others' responses to students' CAM use. The semi-structured protocol allowed the researchers to direct the conversation while leaving space for the participants to add topics that were not covered in the interview protocol..$^{23}$ The focus groups lasted between 45 minutes and one hour and were transcribed.

Data were analyzed using an emergent thematic method of data analysis. ${ }^{23}$ First, the researchers individually read over the focus group transcripts several times to gain a complete understanding of participants' comments. Second, the researchers open-coded the data, identifying repeated patterns and categories that emerged. Third, the researchers then integrated the categories into common themes. After identifying common themes, the researchers highlighted repeated comments representing the themes. Quotations from participants, identified with pseudonyms, are used to illustrate these themes.

\section{Students' communication about complementary/alternative medicine}

\section{Justification of decision making process}

Participants primarily identified two reasons for starting and continuing to use CAM therapies: physical and mental health. Many participants turned to CAM therapies as a way to help alleviate pain resulting from physical ailments or illnesses. In one focus group, Anna discussed how she was diagnosed with mononucleosis. She was prevented from engaging in strenuous exercise but still wanted to be active so she began yoga practice. She explained, At first, I hated them [yoga classes]. They were not fun. My hands hurt. My arms hurt. I didn't get the breathing... But I had to keep moving my body, so I kept coming back for some reason. And then I started to fall in love with it. After recovery, Anna continued her yoga practice; it became such an integral part of her daily life that she completed training to be a yoga fitness instructor and started teaching yoga at the university recreation center. Kara, a student athlete, described how CAM therapies such as acupuncture and chiropractic treatments gave her more energy in her sports performance. I physically felt so much better. I was able to run with less effort. Participants who actively practiced yoga (one of the most commonly practiced therapies among participants) benefitted by acquiring more strength, leaner muscles, and increased flexibility. Participants revealed although they generally started using CAM as a secondary source of treating health, for many it evolved into a primary source of maintaining health.

Most participants began using CAM therapies as a way to aid with their mental health, seeing CAM connecting more to their mental and spiritual well-being than their physical well-being. Sam discussed how his interest in mindfulness led him to begin meditation and yoga practices: Meditation helps me understand why things are happening. Additionally, Sam stated that using CAM therapies has pushed him in a positive direction, and has fa- 
cilitated his ability to overcome frustration. Balance and control were two reoccurring facets of CAM during the focus group. Cassie uses aromatherapy and targeted breathing techniques to help address her anxiety: I just prefer it in a way...I have a lot of anxiety issues. So like, especially breathing, it helps me calm down, come down. And I just don't like taking medicine if I don't have to. Many participants believed their CAM therapies have given them empowerment, strength, control, and a happier, balanced life.

\section{Internal questioning}

Although participants identified as CAM users and a majority use CAM daily, many participants stated they initially struggled with and had concerns about CAM therapies. One of the major reasons for this struggle was because participants were not sure how and if CAM conflicted with their religious beliefs and practices. Sam explained how he struggled with meditation initially because it did not correlate with his religious views. There is a lack of openness due to religious views; I saw it as going against my religion. Sam discussed how he now transforms his religious views with meditation practices as a source of devotion to universal love. Likewise, Abby connects her CAM practice to religion by framing mediation as prayer, stating, Just to know that someone higher than medicine can cure you.

Many participants also discussed how they became discouraged about CAM therapies when they did not see immediate results. Grace explained how she first became discouraged to practice CAM therapies because she was not receiving instant results. She continued her statement by explaining that she had to continuously practice yoga, in order to balance her mental and physical health. In the end, many participants rationalized their decision to continue to use CAM because of the lack of instant success. As Cassie stated, You aren't going to get worse from doing alternative medicine. And it may take a while, but maybe that's healthy.

\section{Communication barriers}

Although the study's participants are active CAM users, they do not actively communicate about CAM or their specific CAM practices. Kevin specified that he only talks openly about his CAM use with one friend, who also actively participates in these therapies. For some participants, this was related to the stigma associated with CAM practices. Anna explained that many of her friends stereotype her due to her CAM practices. They call me a hippie; even though people are starting to accept CAM, they are not open to trying it themselves. For Anna, this is especially difficult because she is a trained fitness instructor and teaches yoga. Although there still remains a stereotype about CAM users, participants generally believed that the stigma is not as great as with other novel practices, but is not widely accepted. As Nadine stated, Some practices like yoga, everyone is okay with. But talk to someone about acupuncture, no. I think they will roll their eyes.

To counter the stigma about CAM, participants identified specific communication strategies when talking about CAM. Most participants strongly emphasized that they do not initiate conversations or bring up CAM during conversations. Madison stated, Unless someone wants to know more about my CAM use, I don't openly advertise $i t$. Participants like Andrew find ways to talk about CAM without discussing some of the alternative or spiritual beliefs connected to those practices. He explained how he does not go into too much detail about his CAM use because he doesn't want to alienate himself from people. He added, If you do discuss CAM, keep it simple and grounded; people understand things conceptually and abstract principles are not visible.

Participants also struggled to communicate with their health care providers about CAM, especially since they see it as an equal part of their daily health practice. Nadine stated, I won't say anything, not unless they [providers] asked. Laura stated that her providers do not talk about CAM therapies, perhaps due to their medical training. Many participants agreed they do not actively discuss their therapies with their providers. Participants stated they never thought to raise the topic during the conversation. Kevin believes doctors as unwilling to communicate with their patients about CAM therapies because they tend to focus on Western medicine that you can test scientifically. Laura also discussed how time constraints limit medical providers from discussing CAM therapies: Instead of taking a holistic approach to heal, many providers just prescribe medicine.

\section{Discussion}

This study used focus group interview data from college students who actively use CAM. Students reported having positive experiences and benefits with their CAM therapies, including having control and balance with their health. This study's finding supports previous research which suggest people use CAM to address physical ailments and mental health ${ }^{11,24}$ and are more likely to have a positive attitude, more self-direction, and more self-control. ${ }^{10}$ Participants also indicated that they do not actively communicate with their providers, family, and friends about their CAM usage. Participants believed their providers do not participate in CAM discussion due to the lack of scientific evidence and integration into the biomedical health care field. ${ }^{25}$

Student participants' decision-making about CAM use was insightful. For many of the participants, CAM use was unexpected and also met with skepticism, in part because CAM was simply a short-term alternative: yoga because a student had mono, using acupuncture to ease pain after surgery. All but one participant came to practice CAM therapies because they genuinely wanted to know 
about the practice. The use of CAM, for the most part, was completely patient-led, ${ }^{26}$ where they arrived to the practice of CAM with little to no previous knowledge about CAM. Instead, they learned about CAM as they practiced. Additionally, the students' enjoyment with CAM therapies helped to transform it from a short-term alternative to a long-term practice. Much of the research on CAM, including those cited in this study, frame CAM as a temporary health practice during the treatment of another health issue. These findings suggest patients quickly move past seeing CAM as temporary treatment and are able to integrate into their everyday health practices.

That being said, this integration is not always seamless. Several participants struggled to reconcile their CAM practices with their religious beliefs. Because many CAM therapies are grounded in enthocultural health practices, ${ }^{27}$ participants were not sure how the tenets of their practice matched with their particular religion. Part of this confusion comes from the fact that many individuals who use CAM therapies do not consider how or why CAM practice is supposed to work. ${ }^{28}$ To do so, would ask individuals to call into question beliefs about greater powers or higher beings. ${ }^{28}$ The findings from this study underscore how participants, even those who have been practicing CAM for longer periods of time, still question how CAM matches with their religious beliefs. Participants in this study appear to reconcile any differences on the tension between religious and

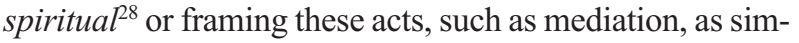
ilar to their religious practices. Participants see CAM truly as complementary to their religious practices.

The findings from this study raise some interesting implications for patient communication about CAM practices. Although CAM use is becoming more popular, participants identified a number of stigmas and stereotypes still associated with CAM use, even more mainstream practices like yoga. The fear of being labelled as weird, a hippie, or one of those people prevents these students from openly talking about CAM practices and using their experiences to educate others. The passive communication strategies employed by participants to counter these stigmas, although helpful, rely on others to be interested, open, and nonjudgmental. Participants were unable to identify successful active communication strategies to dispel negative opinions about CAM. The difficulty or even lack of communication about CAM because of stigmas associated with different CAM therapies could have lasting negative impacts on communication and outcomes. For example, how will family members or friends answer questions about a student's health practices in an emergent care situation? The stigma friends and family associate with CAM could prevent them being able to speak correctly about a student's health practices or be a health advocate for a student.

Participants also indicated that they do not actively communicate with their providers, family, and friends about their CAM usage. Participants believed their providers do not participate in CAM discussion due to the lack of scientific evidence and integration into the biomedical health care field. ${ }^{25}$ The lack of communication about CAM with providers is also a concern because it suggests that these patients are not being honest and open with their providers about their everyday health practices. This is especially concerning because patients could be omitting important information about how they maintain their health. One participant, Megan, who uses a number of herbal supplements, even stated that she only lists multivitamins on her office intake forms because she does not want to be judged by her provider. This can be problematic as there are specific herbal supplements which cause drug interactions..$^{29} \mathrm{CAM}$ users also need to be able to talk to their providers if they are concerned about certain treatment plans, such as mediation. Research suggests that patients who do not agree with treatment plans are less likely to be compliant. ${ }^{30}$ Nonadherence, coupled with patients not feeling they can be honest about their everyday CAM use, could create tension and even adverse events for patients.

\section{Conclusions}

The primary limitation of this study was sample size. It was very difficult to recruit students who consider themselves CAM users or who see the use of certain therapies like yoga as CAM. The sample was also homogenous; all participants attended the same public university and consisted primarily of Caucasian women. It is possible that male college students, students of color, and students from private or religiously affiliated universities use, think, and talk about CAM differently. Future CAM research should also specifically observe students' CAM use for mental and spiritual health. Perhaps these therapies can aid in anxiety and stress reduction in young adults. The findings also underscore how communication about CAM is still a struggle for users, especially with providers. More research is needed about how to improve communication between patients and providers about CAM practices. Additionally, researchers need to examine how students talk to family members about their CAM practices, especially in cases when their parents or other family members are not knowledgeable about or interested in CAM.

A 2018 survey found that while $10 \%$ of US adults have visited a CAM practitioner, $70 \%$ of those same adults believe in the positive effects of CAM practices and $78 \%$ of adults aged 18-29 believe CAM practice is positive..$^{31-32}$ Those are promising numbers for CAM practitioners, patients, and advocates; however, communication and structural barriers still exist which negatively impact patients' ability to practice CAM. Lack of access to CAM providers and lack of health insurance coverage of CAM modalities still inhibit patients in need of care. This can be especially troubling for patients who turn to CAM when allopathic medicine does not offer relief for health issues like chronic pain and fibromyalgia. As CAM therapies become more 
commonplace in everyday health, positive communication strategies will become even more important so patients, providers, friends, and family can have productive conversations about CAM. These successful conversations will help break down stigmas and help individuals embrace the healing powers of complementary and alternative health practices. Improving communication about CAM is a small, but needed step, in order for CAM practices to be accepted by Western medical systems and covered by US health insurance companies as equal to allopathic medicine.

\section{References}

1. Tovey B, Broom A. Oncologists' and specialist cancer nurses' approaches to complementary and alternative medicine and their impact on patient action. Soc Sci Med 2007;64:2550-64.

2. National Center for Complementary and Integrative Health. Complementary, alternative, or integrative health: what's in a name? Available from: https://nccih.nih.gov/health/integrative-health. Accessed: June 2016.

3. Kelner M, Wellman B, Boon H, Welch S. Responses of established healthcare to the professionalization of complementary and alternative medicine in Ontario. Soc Sci Med 2004;59:915-30.

4. Gundling KE. When did I become an 'allopath'? Arch Intern Med 1998;158:2185-6.

5. Zhang Y, Peck K, Spalding M, et al. Discrepancy between patients' use of and health providers' familiarity with CAM. Patient Educ Couns 2012;89:399-404.

6. Ho E, D'Agostino T, Yadegar V, et al. Teaching patients how to talk with biomedical providers about their complementary and alternative medicine use. Patient Educ Couns 2012;39: 405-10.

7. Deng G, Frenkel M, Cohen L, et al. Evidence-based clinical practice guidelines for integrative oncology: complementary therapies and botanicals. J Soc Integr Oncol 2009;7:85-120.

8. Rakel D, Guerrera M, Bayles B, Desai G, Ferrara E. CAM education: promoting a salutogenic focus in health care. $\mathrm{J}$ Altern Complement Med 2008;14:87-93.

9. Lees J, Tovey P. Counseling and psychotherapy, complementary and alternative medicine and the future of healthcare. Br J Guid Counc 2012;40:67-81.

10. Chng C, Neill K, Fogle P. Predictors of college students' use of complementary and alternative medicine. Am J Health Educ 2003;34:267-71.

11. Bishop FL, Yardley L, Lewith GT. A systematic review of beliefs involved in the use of complementary and alternative medicine. J Health Psychol. 2007;12:851-67.

12. LaCaille R, Kuvaas N. Coping styles and self-regulation predict complementary and alternative medicine and herbal supplement use among college students. Psychol Health Med 2001;16:323-32.

13. Kessler RC, Davis RB, Foster DF, et al. Long-term trends in the use of complementary and alternative medical therapies in the United States. Ann Intern Med 2001;135:262-8.

14. Van Der Walt G, Laughharne J, Janca A. Complementary and alternative medicine in the treatment of anxiety and depression. Curr Opin Psychiatry 2008;21:37-42.

15. Johnson P, Ward A, Knutson L, Sendelbach S. Personal use of complementary and alternative medicine (CAM) by US health care workers. Health Serv Res 2012;47:211-27.

16. Richardson MA, Sanders T, Palmer L, et al. Complementary/alternative medicine use in a comprehensive cancer care center and the implications for oncology. J Clin Oncol 2000;18:2505-14.

17. Dogu G, Kargi A, Tanriverdi O, et al. Complementary/alternative medicine experience in cancer patients: a questionnaire-based survey. Int J Hematol Oncol 2014;24:45-53.

18. Druss B, Rosenbeck R. Associations between use of unconventional therapies and conventional medical services. JAMA 1999;283:651-6. Available from: http://jama.jama network.com.

19. Zhang Y, Peck K, Spalding M, et al. Discrepancy between patients' use of and health providers' familiarity with CAM. Pat Educ Couns 2012;89:399-404.

20. Soklardis S, Kelner M, Love R, Cassidy D. Integrative health care in a hospital setting: communication patterns between CAM and biomedical practitioners. J Interprof Care 2009;23:655-67.

21. Humpel N, Jones SC. Gaining insight into the what, why and where of complementary and alternative medicine use by cancer patients and survivors. Eur J Cancer Care 2006; 15:362-8.

22. Ho EY. 'Have you seen your aura lately?': examining boundary-work in holistic health pamphlets. Qual Health Res 2007;17:26-37.

23. Tracy SJ. Qualitative research methods: collecting evidence, crafting analysis, communicating impact. Malden, MA: Wiley-Blackwell; 2013.

24. Johnson S, Blanchard A. Alternative medicine and herbal use among university students. J Am Coll Health 2006;55: 163-8.

25. Shelley BM, Sussman AL, Williams RC, et al. 'They don't ask me so I don't tell them': patient-clinician communication about traditional, complementary and alternative medicine. Ann Fam Med 2009;7:139-44.

26. Evans M, Sharp D, Shaw A. Developing a model of decision-making about complementary therapy use for patients with cancer: a qualitative study. Patient Educ Couns 2012; 89:374-80.

27. Spector RE. Cultural diversity in health and illness. 8th ed. Needham, MA: Pearson; 2013.

28. Brown CG. The healing gods: complementary and alternative medicine in Christian America. New York, NY: Oxford University Press; 2013.

29. Gardiner P, Phillips R, Shaughnessy AF. Herbal and dietary supplement-drug interactions in patients with chronic illnesses. Am Fam Physician 2008;77:73-8.

30. Martin LR, Williams SL, Haskard KB, DiMatteo MR. The challenge of patient adherence. Ther Clin Risk Manag 2005;1:189-99.

31. Statista Survey. Percentage of U.S. adults who have ever tried alternative practitioners as of 2017. New York: StatistaThe Statistics Portal. Available from: https://www-statistacom.libdata.lib.ua.edu/statistics/668542/us-adults-who-evertried-alternative-practitioners-treatments/.

32. Statista Survey. Percentage of U.S. adults believe in positive effects of complementary and alternative medicine (CAM) as of 2017. New York: Statista-The Statistics Portal. Available from: https://www-statista-com.libdata.lib.ua.edu/statistics/667468/us-adults-with-positive-views-on-alternativemedicine-by-age/. Accessed: March 2018. 